\title{
Cumplimiento de las actividades preventivas y de promoción de la salud en un centro de hemodiálisis
}

\author{
Susana Martínez Gómez \\ $M^{a}$ del Carmen Gago Gómez \\ Enrique Gruss Vergara* \\ $M^{a}$ del Mar de Andrés Vózquez \\ Belén Marco García \\ Dolores Piña Simón \\ Alicia González Horna \\ Carmen Gálvez Serrano \\ José Ramón Sánchez Mangas \\ Paloma Hernando Lucio
}

Fundación Renal Iñigo Álvarez de Toledo.

Centro "Los Llanos" Madrid.

*Fundación Hospital Alcorcón. Madrid.

\section{RESUMEN}

Las actividades de prevención y promoción de la salud son abordadas en la población general por el Programa de Actividades Preventivas y de Promoción de la Salud (PAPPS). Los pacientes en hemodiálisis (HD), por su elevada comorbilidad y alteración inmunológica deberían tener un elevado cumplimento de las mismas. El objetivo de nuestro trabajo fue conocer mediante un estudio observacional transversal en 72 pacientes en HD extrahospitalaria el cumplimiento de algunas actividades del subprograma del adulto de los PAPPS.

Resultados. Recomendaciones sobre el estilo de vida: tabaquismo, el 19.4\% fumaban una media de 14.78 cigarrillos/día. Alcohol, el 41.7\% eran consumidores con una media de 106.08+/-87.1 $\mathrm{gr} / \mathrm{semana}$ en varones y 79.6+/-91 gr/semana en mujeres. Programa de prevención del cáncer: cáncer de mama, la media de cumplimiento global fue del 54.4\%; el $42.8 \%$ de forma completa, el $35.7 \%$ de forma incompleta y el $21.4 \%$ no tenía ninguna mamografía. Cáncer de cerviz, la media de cumplimiento global fue 59\%; el 56.2\% con cumplimiento del 100\%, el 43.7\% de forma incompleta y el $18.75 \%$ de pacientes no se había realizado ninguna citología cervicovaginal. Programa de prevención de enfermedades infecciosas: un $19.4 \%$ estaban vacunados frente al neumococo; $22.2 \%$ frente al tétanos-difteria; $73.6 \%$ frente a la gripe y el $97.7 \%$ frente a la Hepatitis B. Actividades preventivas de alteraciones de la visión en los mayores: la media de cumplimiento fue del 30.34\%; el 13.6\% de forma completa; el 52.3\% de forma incompleta y el 34.1 \% no tenía ninguna revisión oftalmológica.

El cumplimiento de las recomendaciones de los PAPPS en HD es bajo. Enfermería debería tomar un papel activo en el incremento de estas actividades.

$\begin{array}{ll}\text { PALABRAS CLAVE: } & \text { PREVENCIÓN } \\ & \text { PROMOCIÓN DE LA SALUD } \\ & \text { ATENCIÓN PRIMARIA } \\ & \text { ENFERMERÍA } \\ & \text { HEMODIÁLISIS }\end{array}$




\section{FULFILMENT OF THE ACTIVITIES OF PREVEN- TION AND HEALTH PROMOTION IN A HAEMO- DIALYSIS UNIT}

\section{SUMMARY}

Prevention and health promotion activities are dealt with in the general population by the Programme of Preventive Activities and Health Promotion (PAPPS). Given their high joint morbility and immunological alteration, patients in haemodialysis (HD) should be highly compliant. The aim of our work was to use a transversal observational study on 72 patients in outpatient HD to see the fulfilment of some activities of the adult sub-programme of the PAPPS.

Results. Recommendations on lifestyle: smoking, 19.4\% smoked an average 14.78 cigarettes/day. Alcohol, $41.7 \%$ were consumers of an average $106.08+/-87.1 \mathrm{~g} /$ week in men and 79.6+/-91 g/week in women. Cancer prevention programme: breast cancer, average overall fulfilment was 54.4\%; 42.8\% completely, 35.7\% incompletely and $21.4 \%$ had no mammography. Cervical cancer, average overall fulfilment was 59\%; 56.2\% fulfilling 100\%, 43.7\% incompletely and $18.75 \%$ of patients had had no kind of cervico-vaginal cytology. Infectious disease prevention programme: $19.4 \%$ were vaccinated against pneumococo; $22.2 \%$ against tetanus-diphtheria; $73.6 \%$ against influenza and $97.7 \%$ against Hepatitis $\mathrm{B}$. Activities to prevent alternations in the vision of the elderly: average fulfilment was 30.34\%; $13.6 \%$ completely; $52.3 \%$ incompletely and $34.1 \%$ had no ophthalmologic revision.

Compliance with the recommendations of the PAPPS in HD is low. Nursing should take an active role in increasing these activities.

\section{KEYWORDS: PREVENTION \\ HEALTH PROMOTION \\ PRIMARY ATTENTION \\ NURSING \\ HAEMODIALYSIS}

\section{INTRODUCCIÓN}

Está demostrada la eficacia de las actividades de prevención, promoción y educación para la salud donde el profesional de enfermería tiene un papel clave. En España, desde Atención Primaria estas acciones son abor- dadas por el Programa de Actividades Preventivas y de Promoción de la salud (PAPPS), puesto en marcha en enero de 1989, donde se indican recomendaciones preventivas, diagnósticas y terapéuticas sobre problemas de salud de elevada prevalencia y gran relevancia social y sanitaria ${ }^{(1)}$. Los pacientes con insuficiencia renal crónica (IRC) presentan un déficit del sistema inmunológico que causa una mayor incidencia de cáncer y de enfermedades infecciosas. Este hecho, junto con el perfil del paciente renal, que ha cambiado en los últimos años hace que los pacientes que inician hemodiálisis (HD) sean cada vez más añosos y con una mayor comorbilidad, haciendo más relevante la aplicación de estos programas. Además, el acudir de forma periódica y continua a nuestras unidades para dializarse debería facilitar llevar a cabo dichas actividades, especialmente por parte de enfermería que es el personal sanitario que más tiempo permanece con el paciente.

El objetivo de nuestro trabajo ha sido conocer, en los pacientes en HD de nuestro centro, el nivel de cumplimiento de las siguientes recomendaciones del subprograma del adulto del PAPPS: recomendaciones sobre el estilo de vida (hábito tabáquico y alcohol), prevención del cáncer de mama y de cérvix, prevención de enfermedades infecciosas y actividades preventivas de alteración de la visión en mayores.

\section{MATERIAL Y MÉTODOS}

Se realizó un estudio observacional transversal descriptivo durante los meses de marzo y abril 2004, en 72 pacientes en HD de un centro extrahospitalario, siendo criterio de exclusión del mismo aquellos que no entendían español. Las características de los pacientes se muestran en la tabla 1.

\begin{tabular}{|l|l|}
\hline $\begin{array}{l}\text { Número de pacientes } \\
\mathbf{n}=\mathbf{7 2}\end{array}$ & $\begin{array}{l}42 \text { varones: } 58,3 \% \\
30 \text { Mujeres: } 41 \%\end{array}$ \\
\hline Diabéticos & $12(16,6 \%)$ \\
\hline Edad & $65,3(35-86)$ años \\
\hline \hline Tiempo en HD & $\begin{array}{l}\text { Media: } 33,8 \pm 24,8 \text { meses } \\
\text { Rango: }(1-111) \text { meses }\end{array}$ \\
\hline
\end{tabular}

Tabla 1. Características de los pacientes

Los datos se recogieron de la historia clínica y a través de la entrevista al paciente y/o familia. Dentro del subprograma del adulto analizamos las recomendaciones del 
PAPPS respecto aquellas actividades que requerían la realización sistemática de pruebas de cribado en la población asintomática y las recomendaciones sobre el estilo de vida. Las actividades preventivas evaluadas junto con las recomendaciones del PAPPS se muestran en la tabla 2.

\begin{tabular}{|c|c|}
\hline $\begin{array}{l}\text { RECOMENDACIONES } \\
\text { SOBRE EL ESTILO DE } \\
\text { VIDA }\end{array}$ & $\begin{array}{l}\text { Tabaquismo: Detección del consumo de } \\
\text { tabaco a personas adultas. } \\
\text { Abuso de alcohol: Detección del consumo } \\
\text { excesivo de alcohol registrando el consumo } \\
\text { en gr./semana. Se considera consumo de } \\
\text { peligroso o de riesgo límites más seguros } \\
\text { como } 170 \text { gr. en el varón y } 100 \text { gr. en } \\
\text { mujeres. }\end{array}$ \\
\hline \multicolumn{2}{|l|}{$\begin{array}{l}\text { PREVENCIÓN DEL } \\
\text { CÁNCER }\end{array}$} \\
\hline CÁNCER DE MAMA & $\begin{array}{l}\text { Se recomienda la realización de una } \\
\text { mamografía cada dos años en mujeres } \\
\text { entre } 50 \text { y } 70 \text { años }\end{array}$ \\
\hline CÁNCER DE CÉRVIX & $\begin{array}{l}\text { Realización de dos test de Papanicolau } \\
\text { anuales y después cada cinco años en } \\
\text { mujeres entre } 35 \text { y } 65 \text { años. }\end{array}$ \\
\hline OTROS CÁNCERES & $\begin{array}{l}\text { No existe evidencia científica para } \\
\text { recomendar el screening en personas } \\
\text { asintomáticas. }\end{array}$ \\
\hline $\begin{array}{l}\text { PREVENCIÓN DE } \\
\text { ENFERMEDADES } \\
\text { INFECCIOSAS }\end{array}$ & $\begin{array}{l}\text { Indicación de vacunaciones de la gripe, } \\
\text { neumococo, tétanos-difteria y hepatitis } B \\
\text { en pacientes inmunodeprimidos ( } \mathrm{IRC} \text { ). }\end{array}$ \\
\hline $\begin{array}{l}\text { ACTIVIDADES } \\
\text { PREVENTIVAS DE } \\
\text { ALTERACIÓN DE LA } \\
\text { VISIÓN EN MAYORES }\end{array}$ & $\begin{array}{l}\text { Valoración de la agudeza visual incluyendo } \\
\text { fondo de ojo, campo visual y presión } \\
\text { intraocular cada dos años en mayores de } \\
65 \text { años y anualmente en diabéticos }\end{array}$ \\
\hline
\end{tabular}

Tabla 2. Recomendaciones del PAPPS.

Todos los datos de los pacientes han sido recogidos en una base de datos tipo Access.

\section{RESULTADOS}

Los resultados obtenidos según los programas del PAPPS estudiados fueron:

Recomendaciones sobre el estilo de vida. El 19.4\% (14 de 72) de los pacientes son fumadores activos, con una media de 14.78 cigarrillos/día (rango de 2-80), mientras que el 44.4\% (32 de 72) nunca ha fumado. El $36.1 \%$ restante son ex-fumadores, de los cuales el 73\% (19 de 26) llevan más de diez años sin fumar. El 41.7\% (30 de 72) de los pacientes refiere consumir alcohol con una media de consumo de $100.8+/-87 \mathrm{gr}$ de alcohol/semana (106.08 +/- 87.1 gr de alcohol/semana en varones y $79.6+/-91$ gr de alcohol/semana en mujeres).
Programa de prevención del cáncer. Cáncer de mama: un $46.6 \%$ (14 de 30) de mujeres presentan criterio de screening del cáncer de mama siendo la media de cumplimiento global del 54.4\%. Un 42.8\% (6 de 14) lo ha realizado de forma correcta en un 100\%. El $35.7 \%$ (5 de 14) lo hizo de forma incompleta y un 21.4\% (3 de 14) no se había realizado ninguna mamografía. Cáncer de cérvix: Un 53.3 \% (16 de 30) de mujeres presentan criterio de screening del cáncer de cérvix siendo la media de cumplimiento total del $59 \%$. Un $56.2 \%$ (9 de 16) lo realizó de forma correcta en un 100\%. El 43.7\% (7 de 16) lo hizo de forma incompleta y un $18.75 \%$ (3 de 16) no se había realizado ninguna citología cervicovaginal.

Programa de prevención de enfermedades infecciosas. Un 19.4\% (14 de 72) de los pacientes están vacunados frente al neumococo. El 22.2\% (16 de 72) están vacunados frente al tétanos-difteria. El 73.6\% (53 de 72) se vacunaron de la gripe y el $97.7 \%$ están vacunados frente a la Hepatitis B (43 de los 44 pacientes con indicación de vacunación).

Actividades preventivas de alteraciones de la visión en los mayores. El $61.1 \%$ (44 de 72) de los pacientes presentan indicación de revisión oftalmológica con una media de cumplimiento del $30.34 \%$. Un $13.6 \%$ (6 de 44) lo ha realizado de forma correcta en un 100\%. El 52.3\% (23 de 44) lo hizo de forma incompleta y un $34.1 \%$ (15 de 44) no habían acudido a revisiones oftalmológicas.

\section{DISCUSIÓN}

Numerosos estudios muestran que la incidencia de cáncer y de enfermedades infecciosas es mayor en nuestros pacientes que en la población generall(2,3). Además la entrada de personas cada vez de más edad en diálisis, con un porcentaje alto de diabéticos, conlleva un aumento de patologías que se añaden al proceso normal de envejecimiento, como son las alteraciones visuales. Por estos motivos se hace necesario que las actividades de prevención y promoción de la salud, indicadas para la población general se realicen, con mayor intensidad si cabe, en los pacientes en programa sustitutivo renal. El programa de PAPPS desde su inicio en 1989 ha contribuido de forma decisiva a la implantación de este tipo de actividades en nuestro país. Sin embargo, muy pocos estudios se han realizado en los pacientes en programa de diálisis. Todos los profesionales que nos dedicamos al cuidado de la salud, y en especial el personal de enfermería, asumen funciones vitales en la prevención y promoción de la salud, herramientas indispensables para el abordaje integral de todas las necesidades del paciente renal. 
Nuestro trabajo analiza algunas de las recomendaciones actuales de los PAPPS observando que casi un 20\% de nuestros pacientes continúan fumando 15 cigarrillos/día de media, sabiendo que el tabaco es el responsable del $30 \%$ de los cánceres ${ }^{(4)}$. Un $41,7 \%$ beben alcohol con una media de 100,8 gramos/semana, y aunque el consumo no alcanza las cantidades consideradas de riesgo (170 gr/semana en el varón y $100 \mathrm{gr} / \mathrm{semana}$ en mujeres) ${ }^{(5)}$, conviene recordar que el consumo de alcohol tiene un efecto cocarcinógeno con el tabaco siendo después de éste, la sustancia psicoactiva que causa mayor número de muertes en España ${ }^{(5)}$.

El cáncer representa uno de los mayores problemas no sólo para la salud pública y la medicina preventiva sino para la ciencia en general. El cáncer de mama es el de mayor incidencia en mujeres y el de cérvix el segundo cáncer femenino más frecuente en la población mundial(6). Es en este colectivo donde las actividades preventivas cobran mayor importancia con el empleo de programas que permiten detectar un tumor maligno antes de que aparezcan signos y síntomas que ya sean clínicamente detectables. A pesar de incluirse en las pruebas pretrasplante la citología y la mamografía, la media de cumplimiento general del programa fue baja: del $54 \%$ en el screening del cáncer de mama y del 59\% en el de cérvix. Hay que resaltar que cerca del $20 \%$ de las mujeres que se dializan en nuestro centro y con criterio de screening de cáncer de mama y cérvix, no se han hecho ninguna mamografía ni citología cervicovaginal, datos que se asemejan a los observados en otros estudios ${ }^{(7)}$.

Las infecciones en los pacientes en diálisis revisten mayor gravedad y a pesar de la baja respuesta que los pacientes con insuficiencia renal crónica manifiestan ante las vacunaciones, se ha demostrado la efectividad de éstas ${ }^{(8)}$. La importancia de las inmunizaciones reside en lograr la prevención de la enfermedad a través de la vigilancia epidemiológica e inmunización efectiva, y no tenemos que olvidar que las vacunas constituyen una de las actividades preventivas más eficientes. Todos nuestros pacientes tienen indicación de vacunación frente a: tétanos-difteria, gripe, neumococo y virus de la hepatitis $\mathrm{B}^{(9)}$. Sin embargo, el porcentaje de vacunación es muy bajo, sólo un 19\% esta vacunado frente al neumococo, un $22 \%$ frente al tétanos-difteria, y un 73,65\% frente a la gripe. La única excepción de vacunación correcta es la hepatitis $\mathrm{B}$ de la que prácticamente todos los pacientes están vacunados (97.7\%) debido a la existencia de controles serológicos periódicos en nuestras unidades, por la repercusión de esta infección que obliga al aislamiento de monitores de HD.

Otro problema actual es la entrada a diálisis de pacientes cada vez más mayores y aunque deterioros funcionales a esta edad, muchas veces no se perciben como enfermedad por considerarse un proceso normal del envejecimiento, se ha comprobado que repercute en la independencia de la persona siendo en nuestro país la discapacidad ocasionada por problemas visuales la más frecuente, después de las osteo-articulares ${ }^{(10)}$. Sin embargo, sólo un $14 \%$ de nuestros pacientes presentan una prevención adecuada de alteración de la visión y más de un 30\% no ha acudido al oftalmólogo. Teniendo en cuenta que, además, diversas enfermedades presentes a esas edades y frecuentes en nuestros pacientes, como la diabetes o enfermedades cardiovasculares, pueden afectar a la visión, el problema es preocupante. Tenemos que concienciarnos de la importancia de prevenir el deterioro visual a través del programa del PAPPS, mediante el cribado de la población anciana y el control de su estado de salud oftalmológica.

En conclusión, a pesar de que los pacientes en HD tienen un alto contacto con personal sanitario, el cumplimiento de las recomendaciones de las PAPPS en nuestro estudio es bajo. Los resultados obtenidos nos deberían alertar a poner en marcha, en los pacientes en hemodiálisis, los programas de prevención, promoción y educación para la salud que no debemos olvidar, son un componente integral de los cuidados de enfermería.

\section{BIBLIOGRAFÍA}

1. Martín Zurro A, Martín Rabadán M, Forés García D. Hacia una conferencia española sobre prevención y promoción de la salud. Atención Primaria 2001; 28 (Supl.2):11-14.

2. Maisonneuve P, Agodoa L, Gellert R, et al. Cancer in patients on dialysis for end-stage renal disease: an international collaborative study. The Lancet 1999; 354:93-98.

3. Krüger S, Seyfarth M, Sack K, Kreft B. Defective inmune respose to tetanus toxois in hemodialysis patients and its association with diphteria vaccination. Vaccine 1999; 17 (9-10):1145-50.

4. Piedrola G, Del Rey J, Domínguez M, y cols. Medicina Preventiva y Salud Pública. Vol. II. Paradigma; 1993. p. 851-866.

5. Robledo T, Ortega R, Cabezas C, Forés D, Nebot M, Córdoba R. Recomendaciones sobre el estilo de vida. Atención Primaria 2003; 32 (supl2):30-44.

6. Cierco P, González J, Melús E, Bellas B, Mur M, Marzo M. Prevención del cáncer. Atención Primaria 2003; 32 (supl2):45-56.

7. Rush H, Neugarten J. Coco M. Women's health issues in dialysis population. Clinical Nephology 2000; 54 (6):455-462.

8. Antonen JA. Hannula PM. Pyhälä R et cols. Adecuate seroresponse to influenza vaccination in dialysis patients. Nephon Sep. 2000; 86 (1):56-61. 